\title{
N-glycosylation profile analysis of Trastuzumab biosimilar candidates by Normal Phase Liquid Chromatography and MALDI-TOF MS approaches ts
}

\author{
Ivan Sanchez-De Melo ${ }^{a}$, Paola Grassi ${ }^{b}$, Francisco Ochoa ${ }^{a}$, Jorge Bolivar ${ }^{a}$, \\ Francisco J. García-Cózar ${ }^{a}, M^{a}$ Carmen Durán-Ruiz ${ }^{a, *}$ \\ aBiotechnology, Biomedicine and Public Health Department, Cadiz University, Spain \\ biopolymer Mass Spectrometry Group, Imperial College, London UK
}

\section{A R T I C L E I N F O}

Available online 21 April 2015

\section{Keywords:}

Monoclonal antibodies

Biosimilars

N-glycosylation

MALDI-TOF MS

Normal Phase Liquid

Chromatography

2AB fluorescent labeling, HILIC

\begin{abstract}
A B S T R A C T
The pharmaceutical market has entered an era in which the production of new therapeutics is being often replaced by "biosimilars", copies of already commercialized products waiting for the patents to expire in order to be distributed in a more competitive and affordable manners. Due to its relevance, the ErbB2-targeted monoclonal antibody Trastuzumab (Herceptin) used as breast cancer therapy is one of the main targets in the production of biosimilars. A major challenge is to produce antibodies with the same or the closest $\mathrm{N}$-glycosylation pattern seen in the commercialized drug. Several factors, such as growing conditions or cell types employed, can determine the final composition and structure of the glycans, significantly affecting the properties of the generated antibodies. Therefore, an appropriate characterization is essential. In the present study, we describe two different but complementary strategies to characterize the N-glycosylation of two biosimilar candidates of Trastuzumab. In the first case, N-glycans are fluorescently labeled and separated by Normal Phase HPLC. Different sugars will elute at different times and can be identified using specific oligosaccharide standards. In the second approach, released glycans are permethylated and analyzed by MALDI-TOF MS, being able to determine the structure because of the differential sugar masses.
\end{abstract}

Biological significance

The characterization of the $\mathrm{N}$-glycosylation sites of therapeutic recombinant monoclonal antibodies (mAbs) is usually one of the most critical and time consuming steps in the developing process of biosimilars or any other glycosylated drug. Herein we describe two different but complementary approaches to characterize mAbs glycosylation patterns, the use of glycan fluorescence labeling coupled to HPLC and MALDI-TOF MS profile analysis. This article is part of a Special Issue entitled: HUPO 2014.

(c) 2015 Elsevier B.V. All rights reserved.

\footnotetext{
it This article is part of a Special Issue entitled: HUPO 2014.

* Corresponding author at: Biomedicine, Biotechnology and Public Health Dpt., Cadiz University, Spain.

E-mail addresses: ivan.sanchez@uca.es (I. Sanchez-De Melo), p.grassi@imperial.ac.uk (P. Grassi), fochoamartin0168@gmail.com (F. Ochoa), Jorge.bolivar@uca.es (J. Bolivar), curro.garcia@uca.es (F.J. García-Cózar), maricarmen.duran@uca.es, maricarmen.duran@gm.uca.es ( $\mathrm{M}^{\mathrm{a}}$. C. Durán-Ruiz).
} 


\section{Introduction}

Therapeutics based on monoclonal antibodies (mAbs) constitute a major target into the actual biopharmaceutical market due to their predictable properties, controlled functions and long circulating life [1]. Murine and chimeric-based mAbs are being replaced by humanized and fully human mAbs. More than 30 different mAb-based therapeutics have been approved for clinical use and many more are currently in research, development, and pre-clinical stages [2]. It is estimated that therapeutic $\mathrm{mAb}$ and their derivatives account for almost $36 \%$ of the biopharmaceuticals under development [3]. The total sales of the top selling blockbuster mAbs were $\$ 63$ billion in 2013, with the ranks of the top 5 selling mAbs remaining unchanged from 2012: Humira ( $\$ 11$ billion), Remicade ( $\$ 9.7$ billion), Rituxan ( $\$ 7.5$ billion), Herceptin ( $\$ 6.5$ billion) and Avastin (6.5 billion) [4]. Currently, Soliris (eculizumab, Alexion) is the most expensive marketed mAb with a price of $\$ 440,000$ per year of treatment. Conversely, due to the expensiveness of production and characterization of $\mathrm{mAbs}$, there is huge interest in the development of biosimilars for the already commercialized innovators, expected to be released to the market in a more affordable manner once the patents expire. It has been estimated that by 2019 approximately $50 \%$ of the market will correspond to off-patent medicines, giving a high market potential for biosimilars. Both innovative products and biosimilars must achieve high levels of quality, efficacy and safety before approval. Thus, biosimilar manufacturers must ensure that their product conforms as closely as possible to the commercialized one, reducing the need for expensive clinical trials and time to reach the market. Therefore, all parties have an interest in performing comprehensive analysis of their products [1].

Most of the current therapeutic mAbs are humanized or human IgGs produced as recombinant glycoproteins in eukaryotic cells (CHO, NSO and Sp2/0 cells among the most frequently used). Many alternative production systems and improved constructs are also being actively investigated [5]. Synthesized IgG molecules are glycosylated at the $\mathrm{C}_{\mathrm{H}} 2$ domains in the -Fc region, with glycans being covalently attached at the Asn297 residue [6]. IgG glycans represent on average only $3 \%$ of the total molecule mass. Despite this low percentage, it is well known the functional relevance of particular glycoforms on immune effector functions [7], thermodynamic stability, potential for immunogenicity [8] and pharmacokinetics [9] of mAbs. In this regard, glycoprotein analysis is essential within the biopharmaceutical industry, because the glycan structure can affect the safety and efficacy of the final products [10]. The actual development of either innovator or biosimilar mAbs considers the glycosylation profile as a critical quality attribute (CQA), being a detailed characterization of $\mathrm{mAb}$ glycosylation a key requirement at the early stages of development. Regulatory authorities require the glycoform profile to be maintained within strict limits [6].

Glycosylation depends on multiple factors like the production system, selected clonal population and manufacturing process, and may be either genetically or chemically engineered [5]. The biopharmaceutical companies have achieved the establishment of specific cell lines that can be expanded in serum- and protein-free media scaled-up to manufacturing levels while maintaining appropriate glycosylation patterns and minimizing immunogenic glycoforms [6]. Methods that allow the production of recombinant mAbs bearing homogeneous oligosaccharides are now becoming available [11]. In any case, an appropriate validation of the glycan structures synthesized is crucial. Glycan analysis represents a major challenge, due to their inherited complexity, lack of chromophore and the existence of various isoforms (both position and linkage) [10]. According to the European Medicines Agency's (EMA) guidelines (EMA/CHMP/BMWP/ 403543/2010) several orthogonal techniques must be used to identify and quantify glycoforms, glycosylation profiling and carbohydrate contents of biosimilar mAbs. Biopharmaceutical glycosylation profiles are most commonly determined on glycans released from the protein backbone either chemically or enzymatically [10]. From this point, several techniques are applied to elucidate the glycan structures found on mAbs. MS approaches such as MALDI-TOF MS or ESI-MS can provide detailed structural information with short analysis times [12]. MALDI-TOF MS is often used as a first step because its unique capacity to generate rapidly information about the nature and diversity of glycans released from native, recombinant glycoproteins or even more complex biological samples [13]. Glycan derivatization prior to MS analysis, e.g. by permethylation, significantly improves the sensitivity of detection of molecular ions, allows simultaneous analysis of neutral and sialylated oligosaccharides in the positive ion mode, and provides predictable fragmentation that gives characteristic "maps" of fragment ions at each amino sugar residue [13]. Structural assignments derived from the MALDI-TOF MS analysis is mainly based on monosaccharide composition, fragmentation by $\mathrm{MS} / \mathrm{MS}$, enzymatic digest sequencing and knowledge of the glycan biosynthetic pathways [14].

As a drawback, MALDI-TOF MS analysis does not allow distinguishing between isobaric glycans. Alternative strategies, like ion-mobility MS or non-MS approaches such as Normal Phase HPLC (NPLC) coupled to fluorescent labeling can overcome this issue. Ideally a combination of both, MS and non-MS approaches provide complete information regarding glycosylation profiles. NPLC of fluorophore labeled glycans gives great sensitivity and can be performed with a range of chromatography phases to give orthogonal separations [10]. For NPLC the hydrophilic interaction liquid chromatography (HILIC) mode is predominantly used to assign structures. Relative scales are produced by running purified glycan standards and samples of interest are compared with standards' chromatographic profiles. In addition, glycan databases are available to compare and assign structures relative to retention times (RT). The most common fluorescent tags used are 2-aminobenzamide (2AB), 2-aminobenzoic acid (2AA) or 2-aminopyridine (2AP). Finally, capillary gel electrophoresis with laser induced fluorescence (CGE-LIF) offers faster analysis times than NPLC, though currently no databases are available to search mobility against structures, and data need to be cross-correlated with either NPLC or MS approaches when developing and validating methods [10]. In the current approach we have focused on the application of NPLC and 
MALDI-TOF MS to determine the glycosylation profile of two biosimilar mAbs of Trastuzumab.

\section{Materials and methods}

\subsection{Reagents}

Ammonium hydrogen carbonate, sodium chloride, iodoacetic acid (IAA), DTT, $\alpha$-cyano-4-hydroxycinnamic acid (HCCA), and hexanes were purchased from Sigma-Aldrich. Glacial acetic acid, acetonitrile, ammonia, chloroform, DMSO, methanol, propan-1-ol, sodium hydroxide pellets and TFA were from Romil. 2,5 dihydroxybenzoic acid (DHB) was from Fluka. Methyl iodide was obtained from Alpha Aesar. Tris(hydroxymethyl)-aminomethane was from Fisher. 3,4-diaminobenzophenone (DABP) was from Acros Organics. All aqueous solutions were prepared using ultrapure (Milli-Q) water.

\subsection{Preparation of $m A b$ samples}

Both IgG1 mAb biosimilars were obtained from two different clones of human-derived cells and purified from the harvested medium using a Protein A column. The final products were obtained in solution form as mAb1 and $\mathrm{mAb} 2$, both at $1 \mathrm{mg} / \mathrm{ml}$ in citrate-Tris $\mathrm{pH}$ 8. Trastuzumab was obtained from providers (Herceptin $®$, Genentech, South San Francisco, CA).

\subsection{Oligosaccharide analysis by NPLC and fluorescence detection}

\subsubsection{Sample preparation}

An amount of $200 \mu \mathrm{g}$ of mAbs were dissolved in $100 \mu \mathrm{l}$ of $0.1 \%$ Rapigest solution (2 $\mathrm{mg} / \mathrm{ml}$ final solution), reduced with $45 \mathrm{mM} \mathrm{DTT}, 30 \mathrm{~min}$ at $60^{\circ} \mathrm{C}$, and alkylated with $10 \mathrm{mM} \mathrm{IAA}$, $20 \mathrm{~min}$, room $\mathrm{T}^{\mathrm{a}}$ in the darkness, in $100 \mathrm{mM}$ ammonium bicarbonate (Ambic). Glycosylated IgGs were digested with trypsin (Promega) (1:20) to reduce proteins to the peptide level and finally $\mathrm{N}$-linked glycans were released by digestion with Peptide -N-Glycosidase F (PNGaseF, New England BioLabs) (1:25), $18 \mathrm{~h}, 37^{\circ} \mathrm{C}$. Following digestion, acetonitrile was added up to $90 \%$ and $\mathrm{N}$-glycans were purified using HILIC microelution SPE in a 96-well format (Waters, Milford, MA, USA) according to manufacturer's guidelines. Briefly, samples were washed several times with 90\% acetonitrile and eluted with $1 \mathrm{mM}$ ammonium Tris citrate in $10 \%$ acetonitrile, fractions were combined and speed-vac dried. Purified N-glycan samples were labeled with $2 \mathrm{AB}$ using a GlycoProfile $2 \mathrm{AB}$ labeling Kit (Sigma, St. Louis, MO, USA) as indicated by manufacturers. Finally, excess of fluorescent $2 \mathrm{AB}$ was removed using HILIC microelution plates as described above.

\subsubsection{HPLC fluorescence analysis}

The $2 \mathrm{AB}$ labeled glycans (10 $\mu \mathrm{g}$ per sample) were further analyzed by NPLC using a HILIC column (XBridge Amide column, $2.5 \mu \mathrm{m} \mathrm{XP,} 3 \times 100 \mathrm{~mm}$, Waters, Milford, MA, USA) at
$60{ }^{\circ} \mathrm{C}$ and detected by an HPLC fluorescence detector (Hitachi LaChrom Elite $\left.{ }^{\circ}\right)$, using excitation $(330 \mathrm{~nm})$ and emission (428 nm) wavelengths.

Separations were performed according to manufacturer's recommendations, using $100 \mathrm{mM}$ ammonium formate $(\mathrm{pH} 4.5)$ as mobile phase A and 100\% acetonitrile as mobile phase B. For glycan elution, the HPLC gradient was carried out as follows: after $45 \mathrm{~min}$ at $25 \% \mathrm{~A}$ and $75 \% \mathrm{~B}$, flow rate $0.5 \mathrm{ml} / \mathrm{min}$, the gradient went to $40 \% \mathrm{~A}$ and $60 \% \mathrm{~B}$ for $1.5 \mathrm{~min}$ and then to $100 \%$ A until minute 48 , remaining at a flow rate of $0.2 \mathrm{ml} / \mathrm{min}$ for two more minutes, returning to $25 \% \mathrm{~A}-75 \% \mathrm{~B}$ for a further 12 min. N-linked glycan structures were assigned to peaks based on comparison to reference structures (2AB-labeled human IgG N-glycans, Prozyme, San Leandro, CA). G0, G1, and G2 refer to complex, neutral, biantennary structures with 0,1 , or 2 terminal galactose residues, while GOF, G1F and G2F are the corresponding core with fucosylated structures [15]. At least three run replicates were obtained for both biosimilars mAb1 and $\mathrm{mAb2}$, and for Trastuzumab. The relative abundance (RA\%) of each glycan in all three samples is expressed as the average of the percentage of total peak area \pm standard deviations $(\mathrm{RA} \pm \mathrm{SD})$. Intra-assay $\mathrm{CVs} \%$ were calculated between replicates for each glycan in all three mAbs. The RA\% of the glycans in the biosimilar mAbs were compared with those annotated for Trastuzumab using two-tailed t-tests. In order to compare the overall glycan profiles of the HPLC chromatograms, RA\% data profiles were compared using the Pearson product-moment correlation coefficient.

\subsection{Oligosaccharide analysis by MALDI-TOF MS}

\subsubsection{Sample preparation}

$100 \mu \mathrm{g}$ of lyophilized and previously purified antibodies was reduced with $500 \mu \mathrm{l}$ DTT $(2 \mathrm{mg} / \mathrm{ml})$ in $0.6 \mathrm{M}$ Tris buffer, $\mathrm{pH} 8.5, \quad 1 \mathrm{~h}, 37^{\circ} \mathrm{C}$ and carboxymethylated with $500 \mu \mathrm{l}$ Iodoacetic acid $(12 \mathrm{mg} / \mathrm{ml})$ in $0.6 \mathrm{M}$ Tris buffer, $\mathrm{pH} 8.5$, $90 \mathrm{~min}$, room temperature, in the dark. Samples were then dialyzed $48 \mathrm{~h}$ at $4{ }^{\circ} \mathrm{C}$ in $50 \mathrm{mM}$ Ambic and lyophilized prior to digestion with $0.3 \mathrm{mg}$ of trypsin $(1 \mathrm{mg} / \mathrm{ml})$ in $50 \mathrm{mM} \mathrm{Ambic}$ at $37^{\circ} \mathrm{C}, 16 \mathrm{~h}$. Trypsin reaction was stopped by heating the samples at $100{ }^{\circ} \mathrm{C}$ and finally with a few drops of $5 \%$ acetic acid. Products were purified by SepPak C18 columns as described [16], in order to separate glycopeptides from nonglycopeptides.

Glycopeptides were concentrated by speed vac, lyophilized and digested with $5 \mathrm{U}$ of PNGase F $37^{\circ} \mathrm{C}, 24 \mathrm{~h}$, for glycans release and separated from peptides by a SepPak C18 purification using sequential elution with $20 \%$ and $40 \%$ propanol. The glycan fraction was concentrated by speed vac and lyophilized. Finally, N-glycans were permethylated with $\mathrm{NaOH}$ as described [17]. Briefly, samples were dissolved in $500 \mu \mathrm{l}$ of a NaOH/DMSO slurry and $500 \mu \mathrm{l}$ of methyl iodide, gently mixed for $20 \mathrm{~min}$ at room temperature. Permethylation was quenched by the addition of a few drops of ice-cold water, followed by $\mathrm{pH}$ neutralization to approximately $\mathrm{pH} 6.5$ with $5 \%$ acetic acid.

Glycans were then extracted with chloroform and purified with sequential fractions of acetonitrile by Sep-Pak C18. Samples were concentrated in a SpeedVac, lyophilized, diluted in $10 \mu \mathrm{l}$ methanol and mixed 1:1 with a DHB matrix 
solution (1 $\mathrm{mg} / \mathrm{ml} \mathrm{80 \%} \mathrm{methanol)} \mathrm{for} \mathrm{further} \mathrm{analysis} \mathrm{by}$ MALDI-TOF MS.

\subsubsection{MALDI-TOF MS analysis}

MALDI-TOF MS analysis was performed on a Voyager-DE sSTR MALDI-TOF (PerSeptive Biosystems, Framingham, MA, USA) in the reflectron mode with delayed extraction. The instrument was calibrated externally using a standard mixture containing des-Arg1-bradykinin (molecular mass 904.46 Da), angiotensin I (molecular mass $1296.68 \mathrm{Da}$ ), human [Glu1]fibrinopeptide B (molecular mass $1570.67 \mathrm{Da}$ ), adrenocorticotropic hormone (ACTH)-(1-17) (molecular mass 2093.08 Da), ACTH-(18-39) (molecular mass 2465.19 Da) and ACTH-(7-38) (molecular mass $3657.92 \mathrm{Da}$ ).

Data were acquired using Voyager 5 Instrument Control Software and were processed using Data Explorer MS processing software from Applied Biosystems. Final spectra were obtained as an average of 500 shots at different laser irradiation positions. Two replicates were obtained per sample. Further MS/MS analyses of peaks observed in the MS spectra were carried out using a 4800 MALDI-TOF/TOF (Applied Biosystems) mass spectrometer. The potential difference between the source acceleration voltage and the collision cell was set to $1 \mathrm{kV}$, and argon was used as collision gas. The 4700 calibration standard kit, calmix (Applied Biosystems), was used as the external calibrant for the MS mode, and [Glu1] fibrinopeptide B human (Sigma) was used as an external calibrant for the MS/MS mode. MALDI spectra were further analyzed using the GlycoWorkbench software suite [18] which allowed assigning glycan masses to the corresponding oligosaccharide structures.

\section{Results}

\subsection{N-linked glycosylation profile of $m A b$ biosimilars by HPLC analysis coupled to fluorescence detection}

$\mathrm{N}$-linked glycans from mAb1, mAb2 and Trastuzumab were released with PNGase F and fluorescently labeled with $2 \mathrm{AB}$ prior to HILIC separation coupled to NPLC analysis. Resultant $\mathrm{N}$-glycan profiles are shown in Fig. 1 whereas RT and abundance (RA\%) of each glycan isoform have been included in Table 1. Four major peaks were observed in the chromatogram of the three mAbs: G0F, G1Fa, G1Fb and G2F, representing $90 \%$ of the total glycan population. G1Fa and G1Fb, two isomeric glycans with the same molecular weight but different oligosaccharide structural distribution, appeared in the chromatogram at different RT $(12.27 \pm 0.08$ and $12.86 \pm$ $0.08 \mathrm{~min}$ respectively). Relative quantification of glycan abundance was also carried out, allowing us to compare between the tested mAbs (Table 1). Thus, while Trastuzumab and mAb2 shared the same pattern of GOF > G1Fa $>$ G1Fb $>$ G2F, the profile seen for $\mathrm{mAb} 1$ was different for the most abundant glycans: G1Fa > GOF > G2F > G1Fb. The rest of the oligosaccharides seen represented less than $10 \%$ of the total glycan population. Finally, although not abundant $(<6.5 \%$ in total for all cases detected), some sialic residues were found in the mAbs (indicated as sial in Table 1, i.e. G1Fa_sia), barely detected in Trastuzumab ( $<1 \%$ in total). In general CV\% values were lower than $10 \%$ but for the less abundant glycans such as the sialylated ones, CV\% could reach $35 \%$.

When samples were compared between themselves, RA\% was quite different between those seen in the biosimilar mAbs and Trastuzumab, with p-values $<0.01$ in most cases (Table 1). Comparison of the glycan profiles of the three mAbs using the Pearson product-moment correlation coefficient indicated that the overall glycan profile of the mAb2 (0.995) was closer to Trastuzumab than mAb1 was to the later (0.965).

\subsection{N-linked glycosylation profile by MALDI-TOF MS analysis}

MALDI-TOF MS analysis of derivatized glycans provided spectra for mAb1 and mAb2 (Fig. 2), with masses correlating to GOF, G1F, and G2F as the most abundant oligosaccharides (see Fig. 3 for glycan nomenclature, molecular weight and correlation with the biantennary structure). The MS spectrum of the glycans linked to Trastuzumab has not being included for simplicity, but it presented the same peaks seen with both mAbs (data not shown). Although MALDI-TOF MS analysis was mostly used to confirm the molecular structures detected by NPLC, the peak intensities from the permethylated glycans indicated a relative abundance similar to the variations seen by liquid chromatography, in agreement with previous reports [19]. Thus, at least for the most abundant glycans, the relative abundance in MALDI (considering peak intensities) and in the NPLC approach (based on fluorescence intensities) appeared as follows: G1F > GOF > G2F in the mAb1 and GOF $>$ G1F $>$ G2F in the mAb2. We could not distinguish between different isoforms of G1F (G1Fa and $\mathrm{G} 1 \mathrm{Fb})$, sharing the same molecular weight. Finally, small signals corresponding to bi- and tri-antennary sialylated glycan residues of m/z: $2401 \mathrm{Da}$ (G1F + sial), $2431 \mathrm{Da}$ (G2 + sial), $2605 \mathrm{Da}$ (G2F + sial) and $3054 \mathrm{Da}$ (triantennary fucosylated with one sialic acid) were detected in the MALDI analysis of the sample mAb2 (not shown in the spectra), but not in the mAb1. This is most likely due to loss of sialic acid during sample handling, as permethylated sialylated glycans are very stable on MALDI and can be observed at very high sensitivity as previously demonstrated [20].

Tandem MS/MS spectra were acquired for some of the glycans detected (i.e. for $\mathrm{m} / \mathrm{z}$ : 1386.92, 2040.02, or $\mathrm{m} / \mathrm{z}$ : 2244.14 Da). Fig. 4 includes an example of MS/MS fragmentation for the glycan G2F (m/z: 2244.14 Da). Structural assignment in both, MS and tandem MS/MS analysis was performed using the GlycoWorkbench suite [18].

\section{Discussion}

In the current work, we applied two alternative but complementary strategies to analyze the $\mathrm{N}$-glycomic profile of two purified mAbs tested as biosimilar candidates for Trastuzumab: NPLC coupled to glycan fluorescent labeling and MALDI-TOF MS. Both methodologies included a first step of enzymatic N-glycan releasing under non-denaturing conditions $[13,14]$ previous to tryptic digestion in order to reduce protein complexity down to the peptide level. This reduction 

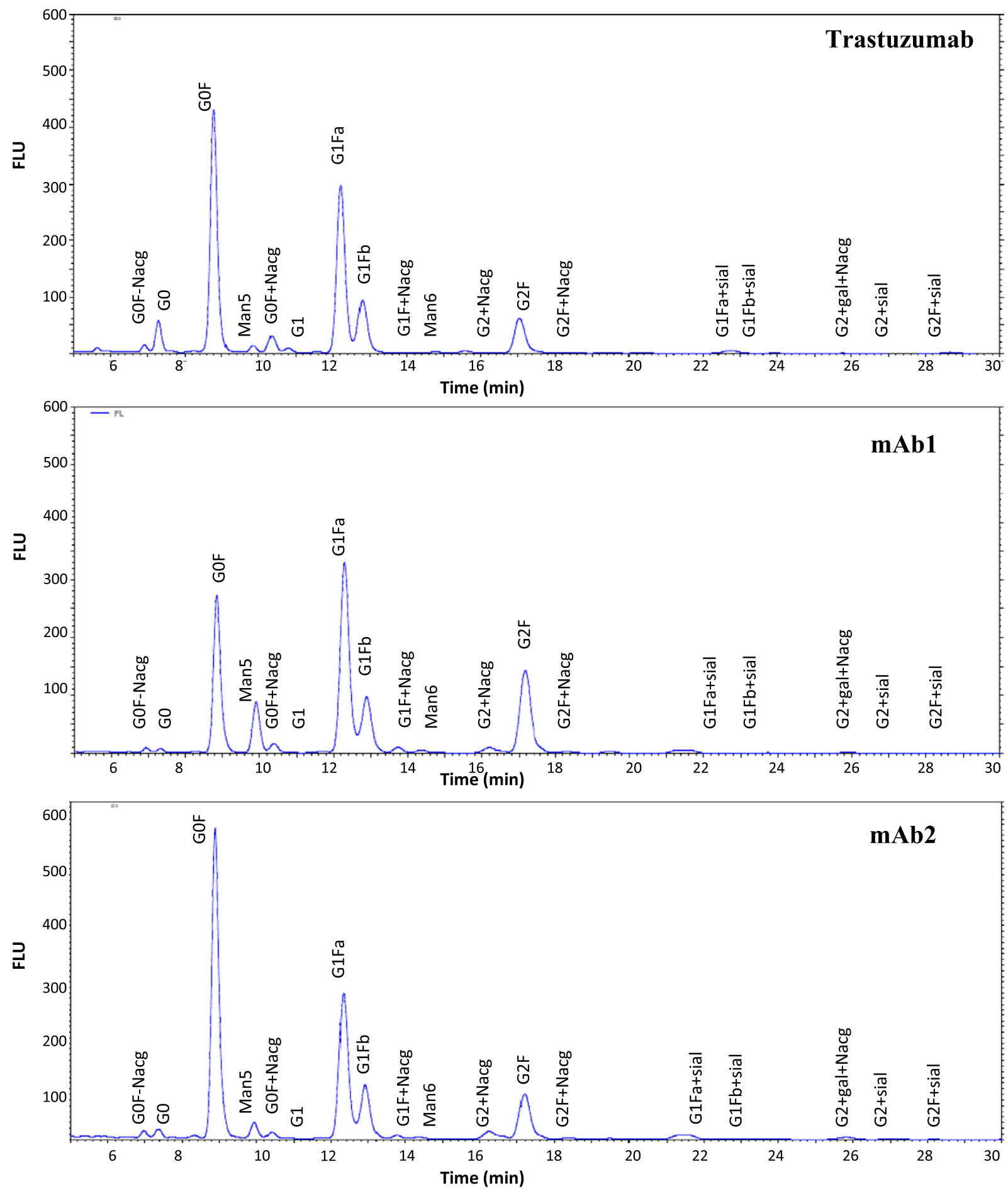

Fig. 1 - N-linked glycan profiles obtained by HPLC analysis from Trastuzumab, mAb1 and mAb2. Glycan assignments were done by comparison with commercially available standards.

in complexity provides better access for the PNGase F to cleave off the intact glycan from the protein side of the antibodies.

Separation and detection of 2AB-labeled glycans by NPLC allowed identifying almost $100 \%$ of the glycan composition in both mAb candidates, including four major glycans (G0, G1Fa, G1Fb and G2F) and 21 minor ones, but no less important oligosaccharides. The identification was done by comparing the elution patterns of the analyzed mAb glycans with commercially available glycan standards. We also distinguished between different isobaric structures, such as G1Fa and $\mathrm{G} 1 \mathrm{Fb}$, due to the slight difference in their respective RT. In the MALDI-TOF MS approach, the most intense peaks correlated to the major glycans (G0, G1 and G2) and several less abundant oligosaccharides were also identified, based purely on the mass of the different carbohydrates, with no 
Table 1 - Percentage of glycan composition from Trastuzumab and mAbs. The retention times (RT) are given in minutes as an average of the RT seen in the three samples, Trastuzumab, $\mathrm{mAb} 1$ and $\mathrm{mAb} 2$, with $\mathrm{CV} \%$ values below $5 \%$ in both interand intra-assays for the RT detected. The relative abundance (RA\%) of each glycan isoform is shown as the calculated area $\% \pm \mathrm{sd}$. CV\% is given for each isoform for all three $\mathrm{mAbs}$ (calculated from the RA\%). The p-values were calculated to compare between the RA\% of Trastuzumab and the biosimilar candidates (Trastuz vs mAb1 and Trast-mAb2). Samples were run in triplicates. N-acetylglucosamine (Nacg); sialic acid (sial); Mannose (Man).

\begin{tabular}{|c|c|c|c|c|c|c|c|c|}
\hline \multirow{2}{*}{$\begin{array}{c}\mathrm{RT}(\mathrm{min}) \\
\text { (average } \pm \mathrm{SD} \text { ) }\end{array}$} & \multicolumn{2}{|c|}{ Trastuzumab } & \multicolumn{2}{|c|}{ mAb1 } & \multicolumn{2}{|c|}{$\mathrm{mAb} 2$} & \multirow{2}{*}{$\begin{array}{l}\text { Trastuz vs. mAb1 } \\
\text { (p-value) }\end{array}$} & \multirow{2}{*}{$\begin{array}{c}\text { Trastuz vs. mAb2 } \\
\text { (p-value) }\end{array}$} \\
\hline & $\mathrm{RA} \%$ & $\mathrm{CV} \%$ & $\mathrm{RA} \%$ & CV\% & $\mathrm{RA} \%$ & $\mathrm{CV} \%$ & & \\
\hline $6.93 \pm 0.04$ & $0,65 \pm 0.05$ & 7.24 & $0.25 \pm 0.01$ & 2.36 & $0.44 \pm 0.03$ & 6.03 & 0.001 & 0.007 \\
\hline $7.31 \pm 0.04$ & $3.62 \pm 0.01$ & 0.16 & $0.22 \pm 0.02$ & 7.03 & $0.54 \pm 0.04$ & 6.91 & $<0.001$ & $<0.001$ \\
\hline $8.83 \pm 0.05$ & $37.73 \pm 0.14$ & 0.37 & $31.68 \pm 0.40$ & 3.46 & $43.61 \pm 1.28$ & 5.92 & $<0.001$ & 0.012 \\
\hline $9.89 \pm 0.05$ & $1.02 \pm 0.02$ & 1.95 & $3.73 \pm 0.11$ & 2.89 & $1.10 \pm 0.05$ & 4.62 & $<0.001$ & 0.118 \\
\hline $10.37 \pm 0.04$ & $2.70 \pm 0.03$ & 1.28 & $0.82 \pm 0.03$ & 3.40 & $0.55 \pm 0.03$ & 4.96 & $<0.001$ & $<0.001$ \\
\hline $10.89 \pm 0.00$ & 0.00 & & 0.00 & & $0.04 \pm 0.00$ & 7.25 & $<0.001$ & 0.220 \\
\hline $11.23 \pm 0.65$ & $0.76 \pm 0.01$ & 1.21 & 0.00 & & $0.13 \pm 0.01$ & 5.38 & $<0.001$ & $<0.001$ \\
\hline $11.48 \pm 0.09$ & $0.19 \pm 0.00$ & 0.37 & $0.19 \pm 0.01$ & 3.34 & 0.00 & & 0.975 & $<0.001$ \\
\hline $12.27 \pm 0.08$ & $31.73 \pm 0.15$ & 0.46 & $37.40 \pm 0.51$ & 2.93 & $32.07 \pm 0.74$ & 6.13 & $<0.001$ & 0.581 \\
\hline $12.86 \pm 0.08$ & $10.90 \pm 0.09$ & 0.79 & $10.68 \pm 0.17$ & 3.08 & $10.95 \pm 0.29$ & 5.94 & 0.044 & 0.823 \\
\hline $13.84 \pm 0.13$ & $0.11 \pm 0.00$ & 0.63 & $0.50 \pm 0.03$ & 6.51 & $0.33 \pm 0.02$ & 4.65 & $<0.001$ & $<0.001$ \\
\hline $14.48 \pm 0.21$ & $0.25 \pm 0.00$ & 0.85 & $0.24 \pm 0.01$ & 5.83 & $0.21 \pm 0.01$ & 6.44 & 0.529 & 0.023 \\
\hline $15.21 \pm 0.45$ & $0.65 \pm 0.00$ & 0.22 & $0.03 \pm 0.00$ & 4.00 & 0.00 & & $<0.001$ & $<0.001$ \\
\hline $16.22 \pm 0.02$ & 0.00 & & $0.47 \pm 0.02$ & 4.22 & $0.48 \pm 0.02$ & 4.52 & $<0.001$ & $<0.001$ \\
\hline $17.12 \pm 0.11$ & $6.36 \pm 0.00$ & 0.04 & $12.30 \pm 0.30$ & 3.18 & $6.46 \pm 0.28$ & 6.33 & $<0.001$ & 0.003 \\
\hline $18.31 \pm 0.08$ & $0.17 \pm 0.01$ & 8.57 & $0.15 \pm 0.01$ & 5.76 & $0.13 \pm 0.02$ & 13.22 & 0.217 & 0.124 \\
\hline $19.47 \pm 0.02$ & 0.00 & & $0.22 \pm 0.01$ & 2.48 & $0.09 \pm 0.01$ & 8.07 & $<0.001$ & $<0.001$ \\
\hline $21.32 \pm 0.01$ & 0.00 & & $0.08 \pm 0.02$ & 19.70 & $0.08 \pm 0.01$ & 12.29 & 0.006 & 0.002 \\
\hline $21.63 \pm 0.04$ & $0.86 \pm 0.03$ & 3.37 & $0.08 \pm 0.02$ & 21.88 & $0.09 \pm 0.01$ & 12.38 & $<0.001$ & $<0.001$ \\
\hline $23.25 \pm 0.51$ & $0.06 \pm 0.00$ & 34.57 & $0.02 \pm 0.00$ & 19.50 & $0.06 \pm 0.01$ & 21.65 & $<0.001$ & 0.919 \\
\hline $24.51 \pm 1.05$ & $0.05 \pm 0.01$ & 2.57 & $0.01 \pm 0.00$ & 28.47 & $0.03 \pm 0.03$ & 82.66 & 0.002 & 0.425 \\
\hline $25.87 \pm 0.04$ & 0.00 & & $0.14 \pm 0.03$ & 23.58 & $0.26 \pm 0.03$ & 11.83 & 0.011 & $<0.001$ \\
\hline $27.48 \pm 0.98$ & $0.34 \pm 0.24$ & 0.38 & $0.01 \pm 0.00$ & 10.10 & $0.03 \pm 0.00$ & 14.29 & 0.282 & 0.343 \\
\hline Total & $100.13 \%$ & & $99.43 \%$ & & $97.68 \%$ & & & \\
\hline
\end{tabular}

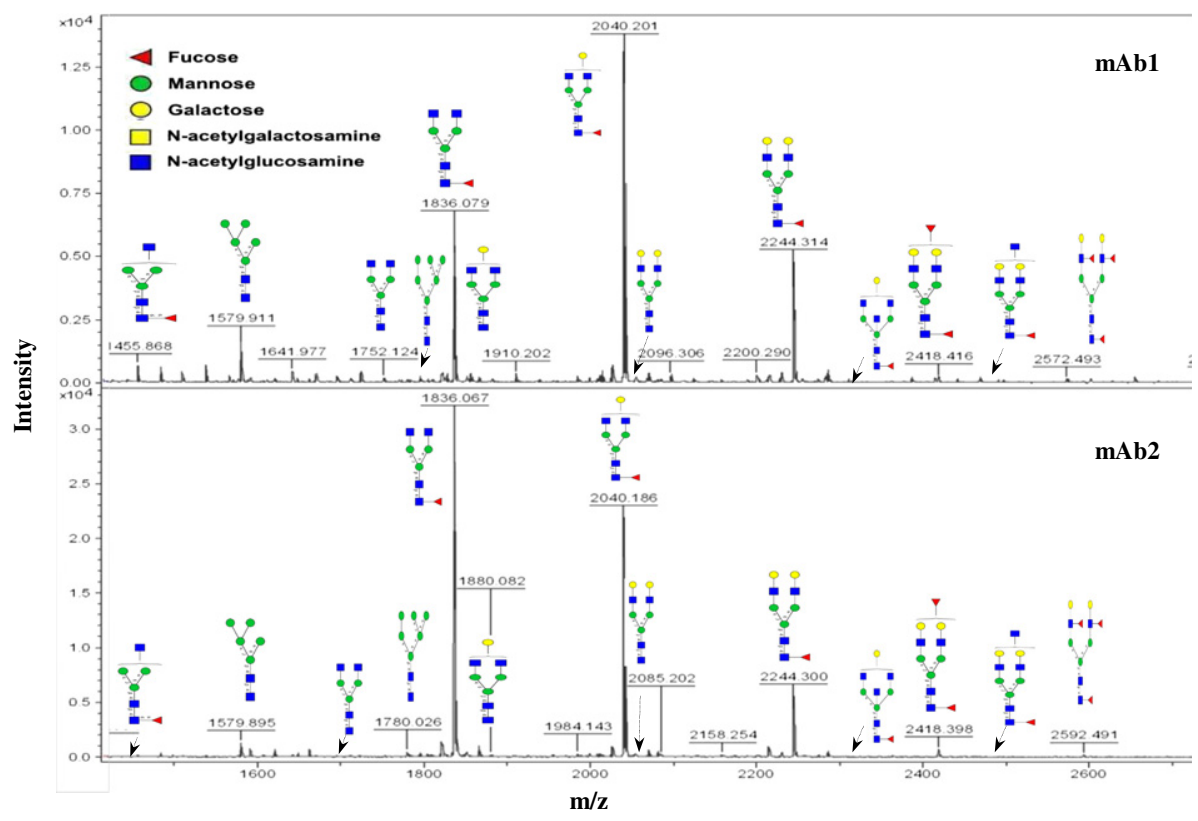

Fig. 2 - Full-scale MALDI-TOF MS spectra of mAb1 and mAb2 glycosylation sites. Structural assignments were based on compositions assigned from molecular masses, complemented by MS/MS information. All molecular ions are $[\mathrm{M}+\mathrm{Na}]^{+}$. Structural assignments are based on monosaccharide composition, fragmentation analyses and knowledge of the glycan biosynthetic pathways. 


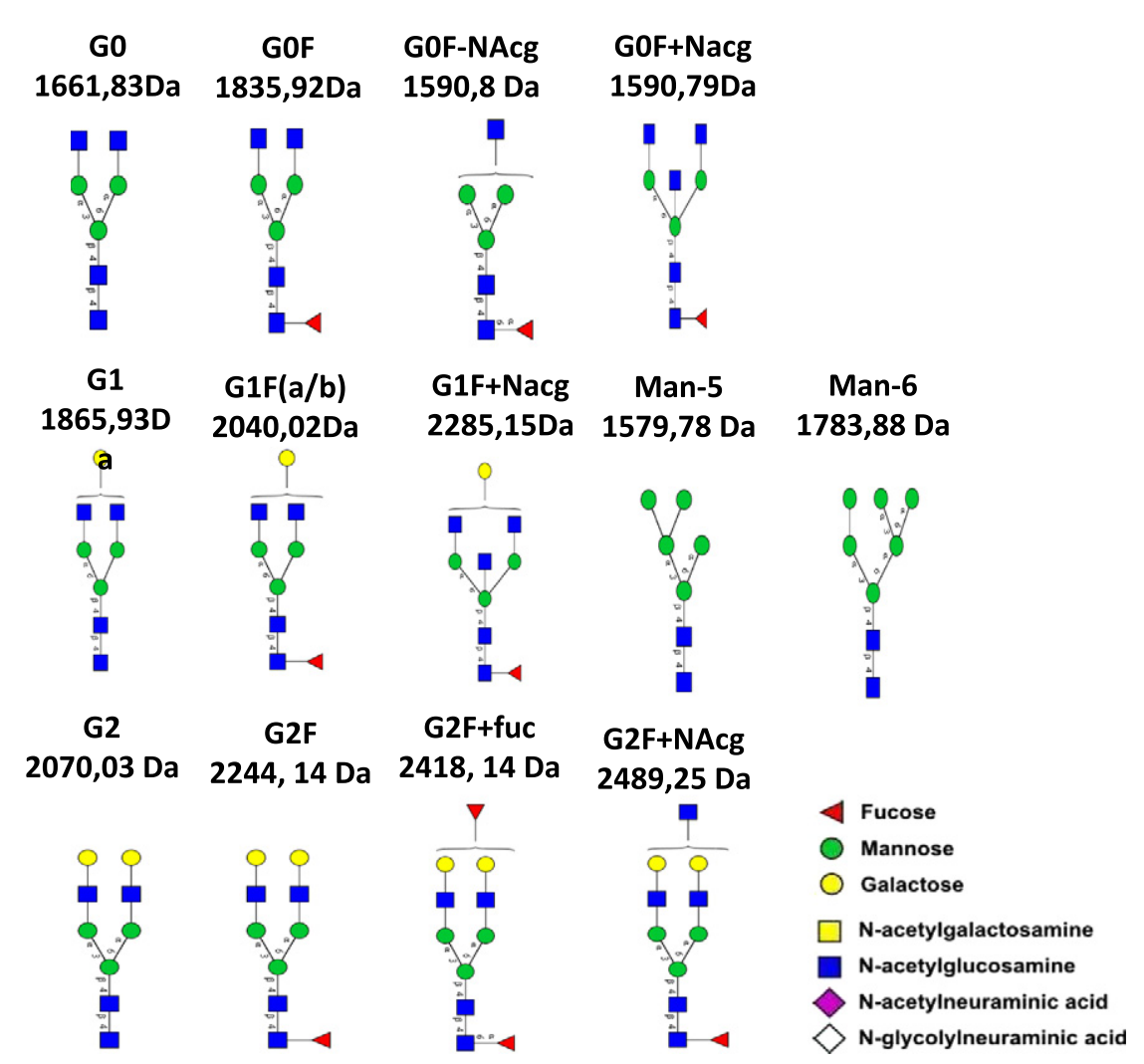

Fig. 3 - Biantennary oligosaccharide structure of the major glycans detected in the study. Sugar symbols are those employed by the Consortium for Functional Glycomics (http://www.functionalglycomics.org).

need for reference standards. Structural assignment was carried out with the support of the GlycoWorkbench suite [18], a software that provides appropriate tools to rapidly assemble and match structure models with MS data and compared to diverse glycan databases to assess the best candidate for the $\mathrm{m} / \mathrm{z}$ evaluated. In contrast to the NPLC

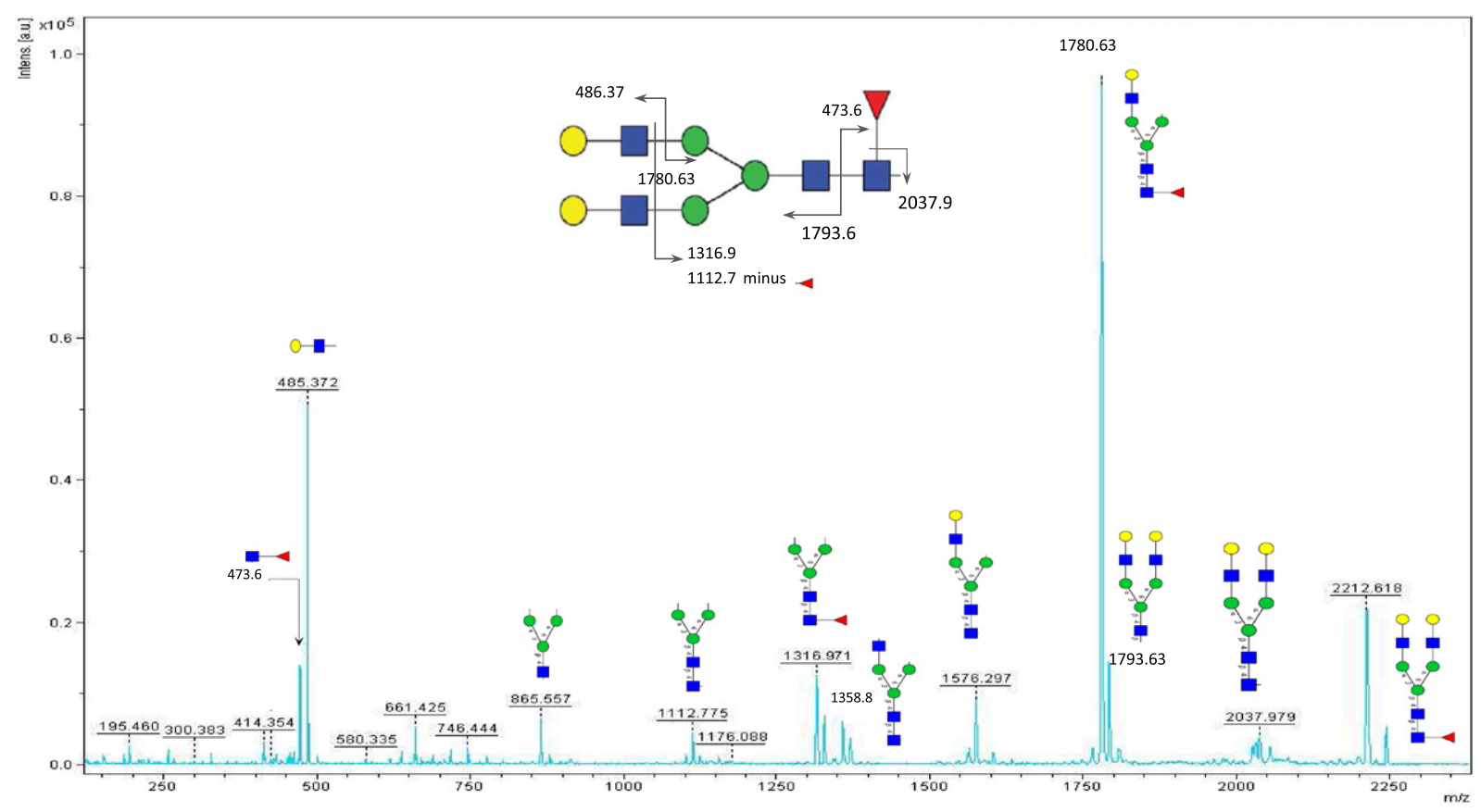

Fig. 4 - MALDI TOF/TOF MS/MS spectrum of the permethylated N-glycan at m/z 2244.14 Da, derived from the glycan spectrum of the mAb1. Assignments of the fragment ions generated are shown. 
approach, the MALDI-TOF MS strategy did not discriminate between isoforms sharing the same molecular weight, although other complementary strategies could be employed in order to elucidate these structures, including further enzymatic treatment and/or linkage analysis [13]. Finally, the use of $2 \mathrm{AB}$ standards in the NPLC approach, and glycan derivatization by permethylation prior to MALDI-TOF MS, significantly helped to detect the less abundant oligosaccharides, including different sialylated forms (comprising less than $2 \%$ each one).

Sialylated glycans are usually difficult to analyze by MALDI-TOF MS and they can easily lose a significant amount of sialic acid in the ion source or after the ion extraction from the ion source. To reduce this loss, sialylated glycans can be analyzed in the linear negative ion mode, which however means that neutral glycans cannot be detected at the same time [13]. In the current approach we used permethylation as derivatization strategy, which stabilizes the negative charge of sialic residues by converting them to methyl esters, thus preventing sialic acid loss while significantly improving the efficiency of positive ion formation [19], allowing us to simultaneously analyze neutral and sialylated oligosaccharides in the ion positive mode.

Permethylation also prevents salt formation, which could complicate the mass spectrum and impair the signal-to-noice ratio for the individual molecular ion species. Furthermore, permethylated oligosaccharides become resistant to insource fragmentation because of the lack of hydroxyl groups which prevents the cleavage of other glycosidic bonds [21]. Herein, small signals corresponding to bi- and tri-antennary sialylated glycan residues were detected in both, NPLC and MALDI-TOF MS approaches (G1F_sial, G2_sial, G2F_sial).

Relative quantification in the NPLC 2AB-fluorescent approach was achieved by comparing, once again, glycan intensities with already known glycan standards. Chromatography of reductively aminated oligosaccharides is generally accepted as a standard method of quantitation, in which the fluorescence correlates with the amounts of individual components [19]. The data presented in Table 1 correspond to the relative abundances of each glycans presented as an average \pm sd of three independent runs per sample, the $\mathrm{mAb}$ biosimilar candidates and Trastuzumab, confirming that the most abundant glycans were the fucosylated species GOF, $\mathrm{G} 1 \mathrm{Fa}, \mathrm{G} 1 \mathrm{Fb}$ and G2F. CV\% values of relative abundances for intra-assays were lower than $10 \%$ in most cases, only higher than $20 \%$ in the less abundant glycans such as the sialylated ones. Comparison of both $\mathrm{mAb}$ candidates with Trastuzumab indicated that the RA\% was not similar to the innovator $(p<0.01)$, although the overall glycan profile of mAb2 was closer to the one seen for Trastuzumab than the glycan profile of mAb1.

Although we initially used MALDI-TOF MS to confirm the molecular structure seen by the NPLC approach by mass assignment, we found that relative peak intensities detected in the glycan spectra were correlated, at least in the most abundant oligosaccharides (GOF, G1F and G2F), with the relative abundances seen by 2AB-LC. It is generally accepted that MS does not allow real quantitation for oligosaccharides unless stable isotope-labeled analogs are incorporated as internal standards [19]. Despite this assumption, several studies have demonstrated that MALDI-TOF MS analyses of permethylated glycans provide reliable relative quantitation information based on signal intensities, particularly when comparisons are made over a small mass range of single ion peaks in the same spectrum [19]. Therefore, even though we decided to take the quantitative data from the NPLC analysis, a more exhaustive processing of the MALDI data could have been done with the permethylated glycans, by measuring the peak height of the monoisotopic ions $[\mathrm{M}+\mathrm{Na}]^{+}$or the integrated peak area for the entire isotopic cluster to obtain a more accurate relative quantitation.

Overall, comprehensive glycosylation profiling confirmed that the proportion of individual glycans was different between the biosimilars and the innovator, although the number and identity of glycans were the same. Our data indicated that at least in the mAbs analyzed, an optimization of the production parameters (i.e. cell growing conditions) appear to be necessary in order to deliver consistent and appropriate glycosylation profiles closer to the innovative drug Trastuzumab. The next logical step, which goes beyond the limits of this work, would be to understand the biological consequences of the glycosylation profiles identified in both biosimilar candidates. Nevertheless, in terms of glycan analysis, both methodological processes described here provided an excellent performance in glycan separation from the protein antibody fraction, delivering quite clean glycan profiles and no cross contamination with the Fc region of the mAbs.

\section{Conclusions}

In this study, we have identified the most and the less abundant N-glycans in two mAbs tested as biosimilar candidates for Trastuzumab. Both methods, NPLC coupled to fluorescence detection and MALDI-TOF MS analysis of permethylated oligosaccharides, have allowed us to identify and confirm the presence of individual glycan molecules, by using standards or by specific mass assignment. In addition, an estimation of the glycan percentage into these antibodies was seen in the NPLC method. In summary, both strategies described herein have provided complementary and supportive data of the N-glycan composition of the mAbs tested, and therefore could be considered as routine methods for the characterization of glycan profiles of recombinant mAbs.

\section{Transparency document}

The Transparency document associated with this article can be found, in the online version.

\section{Acknowledgments}

Part of the work described herein was carried out during the GlycoTRIC training session 2012 (Glycobiology Training, Research and Infrastructure Centre, Imperial College London). Special thanks to Professor Anne Dell, for her critical 
comments and support. The work has been partly funded by the Spanish National Health Institute Carlos III (PI12-02680).

\section{R E F E R E N C E S}

[1] Xie H, Chakraborty A, Ahn J, Yu YQ Dakshinamoorthy DP, Gilar M, et al. Rapid comparison of a candidate biosimilar to an innovator monoclonal antibody with advanced liquid chromatography and mass spectrometry technologies. MAbs Jul-Aug 2010;2(4):379-94.

[2] Thompson NJ, Rosati S, Heck AJ. Performing native mass spectrometry analysis on therapeutic antibodies. Methods Jan 1 2014;65(1):11-7.

[3] Sinclair A. A practical guide to biopharmaceutical manufacturing. Report No.: B-S1-32-6; 2006.

[4] Maggo K. Best selling monoclonal antibody; 2013.

[5] Beck A, Wagner-Rousset E, Bussat MC, Lokteff M, Klinguer-Hamour C, Haeuw JF, et al. Trends in glycosylation, glycoanalysis and glycoengineering of therapeutic antibodies and Fc-fusion proteins. Curr Pharm Biotechnol Dec 2008;9(6): 482-501.

[6] Walsh G, Jefferis R. Post-translational modifications in the context of therapeutic proteins. Nat Biotechnol Oct 2006; 24(10):1241-52.

[7] Shields RL, Lai J, Keck R, O'Connell LY, Hong K, Meng YG, et al. Lack of fucose on human IgG1 N-linked oligosaccharide improves binding to human Fcgamma RIII and antibody-dependent cellular toxicity. J Biol Chem Jul 26 2002;277(30):26733-40.

[8] Kaneko Y, Nimmerjahn F, Ravetch JV. Anti-inflammatory activity of immunoglobulin $\mathrm{G}$ resulting from Fc sialylation. Science Aug 4 2006;313(5787):670-3.

[9] Jones AJ, Papac DI, Chin EH, Keck R, Baughman SA, Lin YS, et al. Selective clearance of glycoforms of a complex glycoprotein pharmaceutical caused by terminal $\mathrm{N}$-acetylglucosamine is similar in humans and cynomolgus monkeys. Glycobiology May 2007;17(5):529-40.
[10] Domann PJ, Pardos-Pardos AC, Fernandes DL, Spencer DI, Radcliffe CM, Royle L, et al. Separation-based glycoprofiling approaches using fluorescent labels. Proteomics Sep 2007; 7(Suppl. 1):70-6.

[11] Jefferis R. Glycosylation as a strategy to improve antibody-based therapeutics. Nat Rev Drug Discov Mar 2009; 8(3):226-34.

[12] Dell A, Morris HR. Glycoprotein structure determination by mass spectrometry. Science Mar 23 2001;291(5512):2351-6.

[13] Morelle W, Michalski JC. Analysis of protein glycosylation by mass spectrometry. Nat Protoc 2007;2(7):1585-602.

[14] Canis K, McKinnon TA, Nowak A, Haslam SM, Panico M, Morris HR, et al. Mapping the N-glycome of human von Willebrand factor. Biochem J Oct 15 2012;447(2):217-28.

[15] Jefferis R. Glycosylation of natural and recombinant antibody molecules. Adv Exp Med Biol 2005;564:143-8.

[16] Dell A. Glycobiology: a practical approach. In: Fukuda MKA, editor. Oxford University Press; 1993. p. 187-222.

[17] Dell A, Reason AJ, Khoo KH, Panico M, McDowell RA, Morris HR. Mass spectrometry of carbohydrate-containing biopolymers. Methods Enzymol 1994;230:108-32.

[18] Ceroni A, Maass K, Geyer H, Geyer R, Dell A, Haslam SM. GlycoWorkbench: a tool for the computer-assisted annotation of mass spectra of glycans. J Proteome Res Apr 2008;7(4):1650-9.

[19] Wada Y, Azadi P, Costello CE, Dell A, Dwek RA, Geyer H, et al. Comparison of the methods for profiling glycoprotein glycans-HUPO Human Disease Glycomics/Proteome Initiative multi-institutional study. Glycobiology Apr 2007; 17(4):411-22.

[20] Antonopoulos A, North SJ, Haslam SM, Dell A. Glycosylation of mouse and human immune cells: insights emerging from N-glycomics analyses. Biochem Soc Trans Oct 2011;39(5): 1334-40.

[21] Lemoine J, Chirat F, Domon B. Structural analysis of derivatized oligosaccharides using post-source decay matrix-assisted laser desorption/ionization mass spectrometry. J Mass Spectrom Aug 1996;31(8):908-12. 